\title{
DOMESTIC DOG OWNERSHIP IN IRAN IS A RISK FACTOR FOR HUMAN INFECTION WITH LEISHMANIA INFANTUM
}

\author{
A. SAMAD MAZLOUMI GAVGANI, HASSAN MOHITE, GHOLAN H. EDRISSIAN, MEHDI MOHEBALI, AND \\ CLIVE R. DAVIES \\ Tabriz University of Medical Sciences, Tabriz, Iran; Department of Medical Parasitology, School of Public Health and Institute of \\ Public Health Research, Tehran, Islamic Republic of Iran; University of Medical Sciences, Tehran, Islamic Republic of Iran; \\ Department of Infectious and Tropical Diseases, London School of Hygiene and Tropical Medicine, London, United Kingdom
}

\begin{abstract}
One explanation proposed for the widespread failure to control zoonotic visceral leishmaniasis by culling infected domestic dogs is that wild canids or humans play significant roles in transmission. The aim of this study was to determine the importance of domestic dogs as the reservoir hosts of visceral leishmaniasis in northwest Iran. A random sample of 3,872 children and 199 dogs in 38 villages was surveyed by the direct agglutination test. Dog ownership details among these households were collected by questionnaire. Parasites isolated from 16 patients and 12 dogs were characterized as Leishmania infantum MON-1. Average seroprevalence in dogs $(21.6 \%)$ was much higher than in children $(7 \%)$. Child seropositivity increased significantly with village dog density in absolute terms $(P<0.001)$ and in relation to $\mathrm{dog} /$ human ratios $(P=0.028)$. Dog ownership within villages also was a significant risk factor for child seropositivity $(P=0.003)$.
\end{abstract}

\section{INTRODUCTION}

Leishmania infantum infections are responsible for visceral leishmaniasis (VL) in at least 70 countries. In contrast to VL caused by Leishmania donovani, the transmission cycle of $L$. infantum is thought to be zoonotic with canids acting as reservoir hosts. In most endemic areas, it is widely believed that domestic dogs (Canis familiaris) are the principal hosts. The main evidence is (1) parasites isolated from dogs are indistinguishable from those in humans, ${ }^{1}$ (2) the infection rates in dogs are relatively high, ${ }^{2}$ (3) there is a correlation between dog and human prevalence, ${ }^{3-5}$ and (4) infected dogs are infectious even if asymptomatic. ${ }^{6-8}$ On this basis, dogs have been the target of large-scale culling programs-notably in China, ${ }^{9}$ Central Asia, ${ }^{10}$ and Brazil. ${ }^{11,12}$ These programs involve the annual serosurveillance of dogs followed by the culling of dogs found seropositive and culling of all stray dogs. The evidence for the success of these programs is limited; for example, in Brazil about 26,000 dogs were culled from 19831997, during which time the annually reported number of human VL cases increased 3-fold. ${ }^{11,12}$ The only 2 reported intervention trials specifically designed to measure the impact of dog culling ${ }^{13,14}$ had inconsistent results. Given this lack of evidence and the unpopularity of the policy among dog owners, there has been a growing call for the policy to be reviewed. ${ }^{15,16}$

One explanation that has been proposed for the apparent failure of the dog culling policy is that domestic dogs may play only a minor role in the transmission cycle. ${ }^{17,18}$ Not only have sylvatic hosts (including foxes, jackals, and opossums) been implicated, but also interhuman transmission has been suggested. ${ }^{18,19}$ The rationale presented for this hypothesis is as follows: (1) Attempts to show a significant correlation between dog ownership and human infection generally have failed, ${ }^{17}(2)$ infection rates of sandflies fed on VL patients are not dissimilar to those fed on infected dogs, ${ }^{18}$ and (3) parasite prevalence in wild canids is often as high as in domestic dogs (Courtenay O, unpublished data).

In this article, we argue that the reason for the apparent failure of dog culling programs is not due to the importance of alternative sources of infection. Using data collected from an endemic zone of VL in northwest Iran, we provide unambigu- ous evidence that domestic dog ownership is a major risk factor for $L$. infantum infection in humans. Domestic dogs should be targeted in VL control programs, where it is costeffective, but alternative tools to culling should be sought.

\section{MATERIALS AND METHODS}

Study population and survey design. The study was carried out in northwest Iran in the neighboring provinces of Ardabil (districts of Meshkin-Shahr and Ghermi) and East Azerbaijan (district of Kalaybar), which together comprise the principal endemic focus of visceral leishmaniasis in Iran. ${ }^{20}$ The average incidence of $L$. infantum infection in this region since 1985 has been about $3 \%$ per year with all ages equally at risk. ${ }^{21}$ Disease symptoms are found only in children, among whom about 1 in 13 infections leads to VL. ${ }^{21}$ The sandfly vectors have not been incriminated conclusively, but suspected vectors found in the focus include Phlebotomus kandelakii and Phlebotomus perfiliewi. ${ }^{22}$ Before this study, no parasites have been isolated and characterized from any nonhuman host of VL in Iran. Serologic and microscopic diagnoses of animals surveyed in endemic areas in Iran have provided evidence, however, for natural Leishmania infections in domestic dogs, golden jackals (Canis aureus), and red foxes (Vulpes vulpes). ${ }^{20,23-25}$

Details of the human survey carried out between August and December 1995 were reported previously. ${ }^{21}$ Informed consent was obtained from parents or guardians, and the study followed the guidelines of the ethical committee at the Tabriz University of Medical Sciences, Iran, and the London School of Hygiene and Tropical Medicine, United Kingdom. Briefly, a random sample of 3,872 children $<10$ years old was surveyed by the direct agglutination test (DAT), comprising $31 \%$ of all the children in 38 villages (17 in Meshkin-Shahr, 10 in Ghermi, and 11 in Kalaybar). Households for the survey were selected by random numbers from a numbered list of all houses in each village (provided by local health workers). Demographic and dog ownership details among the surveyed households were collected by questionnaire. Population data (of humans and dogs) for whole villages were provided by the Ministry of Health. A total of 199 dogs (71 domestic and 128 stray) from the study villages were screened clinically and by 
DAT, and 114 of these additionally were screened parasitologically by microscopic examination of Giemsa-stained spleen and liver aspirates. Parasite isolation attempts were made from 16 human VL patients and from 26 dogs.

Direct agglutination tests. The methodology for human serodiagnoses is standard and has been described previously. ${ }^{21}$ Briefly, antibody titer in finger-prick blood samples collected on filter paper was measured by DAT. The DAT antigen was prepared from log phase promastigotes of a Sudanese strain of L. donovani (strain MHOM/SD/68/1S). The cutoff point to designate human infection with $L$. infantum, 1 in 1,600 , was chosen empirically by seeking the best correlations between the village incidence rates calculated by leishmanin skin test conversion and the village incidence rates calculated by seroconversion (using a series of different DAT titers to define seroconversion). The high specificity of the DAT (97\%) was confirmed by tests on 82 healthy controls (from nonendemic sites) and on 30 patients with other diseases (tuberculosis, toxoplasmosis, and malaria). The sensitivity of the DAT for VL patients was $100 \%$ (22 of 22).

For dog serodiagnosis, dogs first were anesthetized by intramuscular inoculation of acepromazine $(2 \%)$. At least $1 \mathrm{ml}$ of blood was collected from a femur or forearm vein and centrifuged on the same day, and the antibody titer in the serum was measured as for human blood. The cutoff point to designate dog infections ( 1 in 800 ) was chosen to maximize sensitivity (96\%) and specificity (97\%) compared with the results from parasitologic examination of 114 study site dogs, plus an additional 22 parasitologically negative unexposed dogs from a nonendemic region (Tabriz city).

Parasite isolations and characterization. Bone marrow aspirates were obtained by a hospital pediatrician from patients (all children $<5$ years old) with clinical or serologic indications of VL. The aspirates were examined directly for parasites, and the patients were treated if the microscopic diagnosis was positive. Bone marrow aspirates were inoculated into semisloppy Evans' medium and into biphasic NNN medium for growth, isolation, and eventual characterization of Leishmania parasites. For parasite isolation from dogs in the field, liver and spleen aspirates were inoculated into both culture media. For 2 dogs, aspirates also were inoculated into golden hamsters for subsequent reisolation and in vitro cultivation in the laboratory.

Human and dog parasite isolates were taken to London, where they were treated in the same way. Cultures were maintained by serial passaging before mass cultivation in liquid medium ( $\alpha$-MEM with $10 \%$ fetal calf serum). Promastigotes were harvested, washed, resuspended in an enzymestabilizing solution reagent, and lysed before $15-\mu l$ beads were prepared by dropping the supernatant into liquid nitrogen. The isolates were identified by thin-layer, starch-gel electrophoresis using 12 enzymes (ALAT, ASAT, ES, GPI, MDH, MPI, NH, PEPD, PK, 6PGD, PGM, and SOD) as previously reported. ${ }^{26}$ The enzyme profiles were compared with a panel of World Health Organization reference strains including L. infantum, L. donovani, Leishmania tropica and Leishmania major strains.

Statistical analysis. Direct comparisons of prevalence (i.e., between prevalence in dogs and children, between stray and domestic dogs, and between clinically sick and asymptomatic dogs) were carried out by the Yates-corrected chi-square test. Tests of the association between dog ownership patterns and human transmission rate were carried out using the proportion of children $(<10$ years old) who were DAT-positive as the outcome measure of transmission rate. Three parameters were tested for their significance as explanatory variables for child seroprevalence: (1) the population size of dogs in a village, (2) the ratio of dogs to humans in a village, and (3) whether a household owns a dog. We tested the epidemiologic impact of dogs at 2 spatial levels - the village level and the household level. The significance of any association was tested by logistic regression in STATA 7 (i.e., assuming binomial errors on the outcome proportion). In the first 2 analyses, we clustered the data ( $\mathrm{n}=3,872)$ by village $(\mathrm{n}=38)$. In the third analysis, we clustered the data by household $(\mathrm{n}=1,724)$ but included village as an explanatory variable in the model (i.e., we controlled for any intervillage differences in transmission rate). Three villages (nos. 12, 19, and 20) were excluded from this analysis because they contained no seropositive children (Table 1).

TABLE 1

Child seroprevalence in 38 Iranian villages in relation to village dog population, human population, and dog ownership behavior

\begin{tabular}{lcccr}
\hline & & & \multicolumn{2}{c}{$\begin{array}{c}\text { DAT prevalence in children } \\
\text { (no positive/total) }\end{array}$} \\
\cline { 5 - 5 } $\begin{array}{c}\text { Village } \\
\text { code }\end{array}$ & $\begin{array}{c}\text { Dog } \\
\text { population }\end{array}$ & $\begin{array}{c}\text { Human } \\
\text { population }\end{array}$ & $\begin{array}{c}\text { Households } \\
\text { with dog }\end{array}$ & $\begin{array}{c}\text { Households } \\
\text { without dog }\end{array}$ \\
\hline 1 & 65 & 3,559 & $10.5(2 / 19)$ & $2.3(2 / 88)$ \\
2 & 48 & 993 & $6.5(2 / 31)$ & $1.8(1 / 56)$ \\
3 & 51 & 1,271 & $11.8(2 / 17)$ & $3.6(2 / 56)$ \\
4 & 72 & 773 & $25(8 / 32)$ & $18.2(6 / 33)$ \\
5 & 90 & 2,095 & $12.3(7 / 57)$ & $11.1(6 / 53)$ \\
6 & 45 & 2,154 & $3.2(1 / 31)$ & $1.4(1 / 72)$ \\
7 & 97 & 1,518 & $14.8(9 / 61)$ & $10.3(3 / 29)$ \\
8 & 87 & 2,451 & $2.5(2 / 80)$ & $2.9(2 / 79)$ \\
9 & 92 & 4,255 & $9.8(8 / 82)$ & $5.3(5 / 64)$ \\
10 & 37 & 780 & $6.1(2 / 33)$ & $3.7(2 / 54)$ \\
11 & 45 & 201 & $9.1(2 / 22)$ & $0(0 / 19)$ \\
12 & 50 & 1,091 & $0(0 / 26)$ & $0(0 / 50)$ \\
13 & 55 & 1,332 & $2.1(1 / 35)$ & $3.2(2 / 63)$ \\
14 & 38 & 1,047 & $0(0 / 34)$ & $7.4(4 / 54)$ \\
15 & 78 & 4,151 & $10.2(8 / 78)$ & $16.3(8 / 49)$ \\
16 & 40 & 755 & $9.4(3 / 32)$ & $3.3(2 / 60)$ \\
17 & 40 & 581 & $5.4(3 / 56)$ & $2.6(1 / 38)$ \\
18 & 32 & 1,024 & $1.8(1 / 55)$ & $0(0 / 27)$ \\
19 & 36 & 381 & $0(0 / 22)$ & $0(0 / 61)$ \\
20 & 47 & 1,170 & $0(0 / 29)$ & $0(0 / 52)$ \\
21 & 68 & 351 & $6.1(4 / 66)$ & $4.8(3 / 62)$ \\
22 & 89 & 1,176 & $12.5(15 / 120)$ & $4.9(3 / 61)$ \\
23 & 43 & 1,416 & $6.0(7 / 116)$ & $6.3(3 / 48)$ \\
24 & 35 & 686 & $5.5(3 / 55)$ & $5.0(1 / 20)$ \\
25 & 30 & 476 & $8.9(9 / 101)$ & $6.3(3 / 48)$ \\
26 & 69 & 572 & $17.3(22 / 127)$ & $6.4(3 / 47)$ \\
27 & 25 & 246 & $4.8(1 / 21)$ & $0(0 / 27)$ \\
28 & 78 & 2,489 & $8.9(12 / 135)$ & $3.4(3 / 87)$ \\
29 & 137 & 453 & $9.8(5 / 51)$ & $21.4(3 / 14)$ \\
30 & 30 & 994 & $8.1(3 / 37)$ & $4.9(2 / 41)$ \\
31 & 60 & 1,016 & $9.6(8 / 83)$ & $8.9(4 / 45)$ \\
32 & 105 & 833 & $11.9(10 / 84)$ & $12(3 / 25)$ \\
33 & 50 & 623 & $12.9(8 / 62)$ & $0(0 / 14)$ \\
34 & 33 & 139 & $6.4(3 / 47)$ & $13.3(2 / 15)$ \\
35 & 30 & 1,369 & $2.7(2 / 75)$ & $0(0 / 35)$ \\
36 & 42 & 331 & $10.3(6 / 58)$ & $0(0 / 40)$ \\
37 & 69 & 179 & $19(8 / 42)$ & $33.3(2 / 6)$ \\
38 & 64 & 177 & $6.3(3 / 48)$ & $0(0 / 20)$ \\
Total & 2,202 & 45,108 & $8.8(190 / 2,160)$ & $4.8(82 / 1,712)$ \\
\hline & & & &
\end{tabular}




\section{RESULTS}

Infection rate in dogs. Of the 114 study-site dogs examined, 26 were parasitologically positive. Using 1 in 800 as the cutoff point, 25 of 26 parasite-positive dogs were DAT-positive (the single false-negative having a titer of 1/400), and 107 of 110 parasite-negative dogs (i.e., including the 22 unexposed dogs from Tabriz city) were DAT-negative ( 2 false-positives with a titer of $1 / 800$ and one with 1/1,600). The overall seroprevalence in the 199 dogs tested was $21.6 \%$, which was significantly higher than in children $(7 \% ; 272$ of 3,872 ; chi-square $=$ 54.4, $P<0.001)$. Seroprevalence was significantly higher among strays (34 of 128) than among domestic dogs (9 of 71; chi-square $=4.41, P<0.05$ ). Parasite prevalence (chi-square $=33.6, P<0.001$ ) and seroprevalence (chi-square $=25.1$, $P<0.001)$ were significantly greater in dogs with $\geq 1$ clinical symptoms (24 of 47 [51\%] and 23 of 47 [49\%]) than among asymptomatic dogs (2 of 67 [3\%] and 20 of 152 [13.2\%]). The most common clinical symptoms detected were hair loss, weight loss, and overgrowth of toe nails, but dogs were most likely to be parasite-positive or DAT-positive if their clinical symptoms included hepatomegaly or splenomegaly.

There were 19 isolates obtained from the 26 parasitologically positive biopsy specimens from dogs, but 3 were lost to contamination, leaving 16 isolates for characterization (5 from Meshkin-Shahr, 3 from Ghermi, and 8 from Kalaybar)all were identified as $L$. infantum zymodeme LON-49 (equivalent to MON-1). From the 16 bone marrow aspirates taken from human patients, 12 isolates were obtained (of which 7 also were positively diagnosed parasitologically by microscopy): 6 from Meshkin-Shahr, 2 from Ghermi, and 4 from Kalaybar. All isolates were indistinguishable from $L$. infantum World Health Organization reference strain zymodeme LON-49 (i.e., the same as the 16 dog isolates and the only 3 human isolates from the region previously characterized). ${ }^{20}$

Dog ownership and population size as risk factors. The mean dog population size in a village was 58 (range, 25-137), the mean dog/human ratio was 0.049 (range, 0.018-0.39), and $48 \%$ (835 of 1,724$)$ of surveyed households owned a dog. Seroprevalence in children was significantly higher in villages with greater dog populations (odds ratio [OR], 1.014; 95\% confidence interval [CI], 1.01-1.02; $z=4.90, P<0.001)$ and in villages with greater dog/human ratios (OR, 10.9; 95\% CI, 1.3-92; $z=2.19, P=0.028$ ) (Figure 1, Table 1). The odds of child infection increased on average by $14 \%$ for every increase of 10 in the village dog population and by $9 \%$ for every increase of 0.1 in the village ratio of dogs to humans. In the final analysis, we tested whether there was any additional risk to households in owning a dog (other than the mass effect it has on increasing the dog population in a village). Seroprevalence in children was significantly higher in households that owned a $\operatorname{dog}(\mathrm{OR}, 1.54 ; 95 \% \mathrm{CI}, 1.16-2.04 ; z=$ 2.97, $P=0.003)$.

\section{DISCUSSION}

Our results show that L. infantum $\mathrm{MON}-1$ is responsible for leishmaniasis in humans and dogs in northwest Iran. We also showed that seroprevalence in dogs was about 3 times higher than in children, indicating that sandfly biting rates are much higher on dogs than on humans (assuming the 2 species are
A
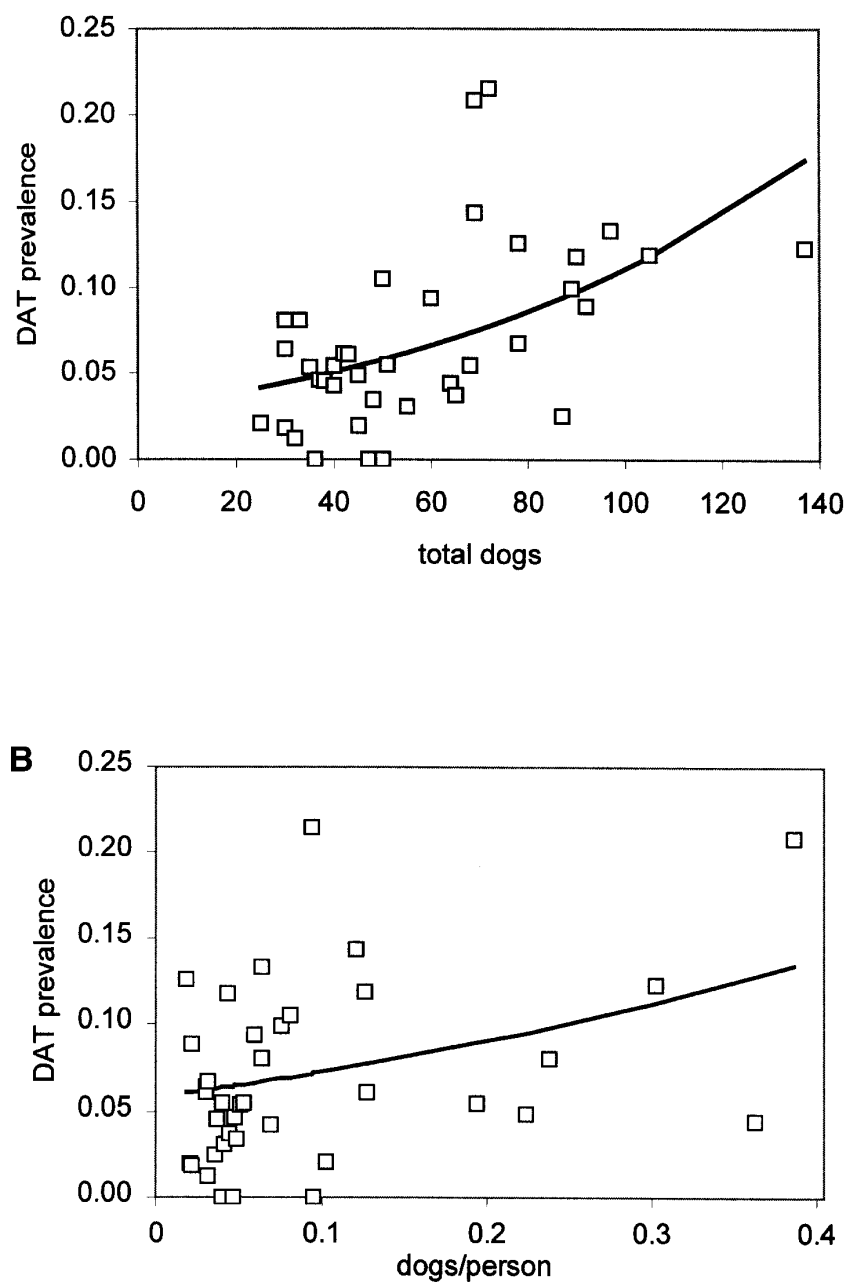

FIGURE 1. The relationship between DAT prevalence in children from 38 Iranian villages versus (A) the total number of domestic dogs in the same villages and (B) the ratio of dogs to persons in the same villages. Squares represent the data from individual villages, and the lines were fitted by maximum likelihood (see text).

equally susceptible and that serorecovery rates are comparable). Neither of these findings proves that dogs are not just accidental hosts similar to humans. We also showed, however, that human infection rates increase with dog density and that owning a dog is a significant risk factor for members of households. The effect of dog density was tested in absolute terms (i.e., population size) and in relation to human density because the former may be confounded by human population size. Both tests were significant. To our knowledge, this is the first study of $L$. infantum to show the epidemiologic significance of geographic variability in dog population size. This is a key prediction of the hypothesis that domestic dogs are the principal reservoir host of VL.

The positive association between dogs and risk to humans is not because increasing the dog population should increase sandfly biting rates on humans; rather it could lead to a reduction in human biting rates if sandflies are diverted from feeding on humans onto dogs. Instead, we propose that dog density affects local transmission rates to humans by increasing the prevalence of infection among sandflies, so increasing 
the entomologic inoculation rate. This increase is not only because dogs have a relatively high prevalence, but also because all studies published to date show that they are relatively infectious compared with other infected hosts, including humans. The infectiousness of dogs, with and without symptoms, has been measured in at least 13 reported studies (Courtney O, unpublished data) with a mean infection rate of $25 \%\left(1,979\right.$ of 7,927 sandflies). ${ }^{7,27,28}$ In contrast, only $8 \%(112$ of 1,365$)$ of sandflies fed on VL patients were infected, ${ }^{8,18,29,30}$ and none of about 2,000 sandflies fed on subclinical cases or cured VL patients became infected. ${ }^{18}$

Previous attempts to test the role of dogs focused on household dog ownership patterns. We are aware of 4 previous studies of $L$. infantum that failed to find a significant epidemiologic association with dog ownership: in Honduras, ${ }^{31}$ Egypt, ${ }^{32}$ and Brazil. ${ }^{17,33}$ Among these studies, the 3 that reported their data found that infection (or VL) rates tended to be higher in households with dogs. A fifth study, in Egypt, ${ }^{34}$ did find a significant epidemiologic association with dog ownership: Seroprevalence in children living in dog-owning households was $5.9 \%$ (11 of 188 ) compared with $1.9 \%$ (7 of 374 ) in households without dogs (OR, 3.3; 95\% CI, 1.2-9.5). Given that sandflies can disperse readily between houses and that dogs also move around and may infect sandflies far from their owners' household, we would expect any potential epidemiologic impact at the household level to be hard to detect. It is thus surprising that 2 of 6 published studies (including this one) have found that dog ownership is a significant risk. Although the only 2 significant results so far detected both come from the Old World, there is no obvious reason why the results should not apply to the New World. The relative role of sylvatic canids in the transmission cycle is likely to be greater in the Old World, where L. infantum originated, rather than the New World, where the parasite is thought to have been introduced with domestic $\operatorname{dogs}^{35}$ and where there is no ancestral wild transmission cycle. All 5 published studies that presented their results show the same positive trend. This consistency adds further weight to the argument that domestic dogs are the principal reservoir host of L. infantum. We cannot discount the possibility, however, that dog ownership is a confounder for some other behavioral factor with a causal association with the risk of infection; for example, ownership of a sheepdog may be associated with farming activities, which could increase the risk of sandfly-human contact.

In conclusion, we argue that the reason for the failure of dog culling programs to control human visceral leishmaniasis is not because dogs are only a minor reservoir host. The explanation instead lies in the following observations: (1) Infected dogs may not yet have seroconverted when surveyed by the Ministry of Health, and these infected dogs may become infectious before the following survey; (2) not all dogs in the endemic zone are surveyed; (3) there is a significant lag between detection of an infected dog and its being culled, and not all such dogs do get culled; and (4) culled dogs soon get replaced by susceptible puppies. Most of these problems are probably intractable, and so alternative control tools for targeting domestic dogs should be sought. ${ }^{36,37}$

Acknowledgments: We are grateful to David Evans for setting up the project, to Paul Coleman and Diarmid Campbell-Lendrum for comments on the manuscript, and to Orin Courtenay for providing permission to refer to unpublished data from his $\mathrm{PhD}$ thesis (London University, 1998): The epidemiology and control of canine visceral leishmaniasis in Amazon Brazil.

Financial support: This work was supported by the Iranian Ministry of Health.

Authors' addresses: A. Samad Mazloumi Gavgani, Hassan Mohite, Tabriz University of Medical Sciences, Daneshgah Street, PO Box 51657, Tabriz, Iran, School of Hygiene and Tropical Medicine, Keppel Street, London, WC1E 7HT, UK, Fax: 98-411-334-7345. Gholan H. Edrissian, Mehdi Mohebali, Department of Medical Parasitology, School of Public Health and Institute of Public Health Research. Tehran University of Medical Sciences, Tehran, Islamic Republic of Iran. Clive R. Davies, Department of Infectious and Tropical Diseases, London School of Hygiene and Tropical Medicine, Keppel Street, London, WC1E 7HT, UK, Telephone: 44-207-927-2350, Fax: 44-207-927-2164.

Reprint requests: Clive Davies, Department of Infectious and Tropical Diseases, London School of Hygiene and Tropical Medicine, Keppel Street, London, WC1E 7HT, UK, Telephone: 44-207-927-2350, Fax: 44-207-927-2164, E-mail: clive.davies@lshtm.ac.uk

\section{REFERENCES}

1. Jimenez M, Ferrer Dufol M, Canavate C, Gutierrez-Solar B, Molina R, Laguna F, Lopez-Velez R, Cercenado E, Dauden E, Blasquez J, 1995. Variability of Leishmania (Leishmania) infantum among stocks from immunocompromised, immunocompetent patients and dogs in Spain. FEMS Microbiol Lett 131: 197-204.

2. Quinnell RJ, Courtenay O, Garcez L, Dye C, 1997. The epidemiology of canine leishmaniasis: transmission rates estimated from a cohort study in Amazonian Brazil. Parasitology 115: 143-156.

3. Tagizade TA, Gasanzade GB, Safyanova VM, Shalmiev GB, Gadzibekova EA, Savina MA, Alieva KK, Faramozov AZ, Gamidov AA, 1989. Visceral leishmaniasis in the Ordubad District of the Nakhichevan ASSR. Meditisinskiya Parasitologiya i Parasitarnye Bolezni 3: 22-27.

4. Cunha S, Freire M, Eulalio C, Critosvao J, Netto E, Johnson WD, Reef SG, Badaro R, 1995. Visceral leishmaniasis in a new ecological niche near a metropolitan area of Brazil. Trans $R$ Soc Trop Med Hyg 89: 155-158.

5. Acedo Sanchez C, Martin Sanchez J, Velez Bernal ID, Sanchis Marin MC, Louassini M, Maldonado JA, Morillas Marquez F, 1996. Leishmaniasis eco-epidemiology in the Alpujarra region (Granada Province, southern Spain). Int J Parasitol 26: 303310.

6. Molina R, Amela C, Nieto J, San-Andes M, Gonzalez F, Castillo JA, Lucientes J, Alvar J, 1994. Infectivity of dogs naturally infected with Leishmania infantum to colonized Phlebotomus perniciosus. Trans R Soc Trop Med Hyg 88: 491-493.

7. Gradoni L, Maroli M, Gramiccia M, Mancianti F, 1987. Leishmania infantum infection rates in Phlebotomus perniciosus fed on naturally infected dogs under antimonial treatment. Med Vet Entomol 1: 339-342.

8. Sherlock IA, 1996. Ecological interactions of visceral leishmaniasis in the State of Bahia, Brazil. Mem Inst Oswaldo Cruz 91: 671-683.

9. Xu Z-B, 1989. Present situation of visceral leishmaniasis in China. Parasitol Today 5: 224-228.

10. Sergiev VP, 1979. Epidemiology of leishmaniasis in the USSR. In Lumsden WHR, Evans DA, eds. Biology of the Kinetoplastida. Vol II. New York: Academic Press: 197-212.

11. Lacerda MM, 1994. The Brazilian leishmaniasis control program. Mem Inst Oswaldo Cruz 89: 489-495.

12. Vieira JB, Coelho GE, 1998. Leishmaniose visceral o calazar: aspectos epidemiologicos e de controle. Rev Soc Bras Med Trop 31(suppl II): 85-92.

13. Dietze R, Barros GA, Teixeira L, Harris J, Micelson K, Falqueto A, Corey R, 1997. Effect of eliminating seropositive canines on the transmission of visceral leishmaniasis in Brazil. Clin Infect Dis 25: 1240-1242.

14. Ashford DA, David JR, Freire M, David R, Sherlock I, Eulalio MC, Samaio DP, Badaro R, 1998. Studies on the control of 
visceral leishmaniasis: impact of dog control on canine and human visceral leishmaniasis in Jacobina, Brazil. Am J Trop Med Hyg 59: 53-57.

15. Tesh RB, 1995. Control of zoonotic visceral leishmaniasis: is it time to change strategies? Am J Trop Med Hyg 52: 287-292.

16. Dye C, 1996. The logic of visceral leishmaniasis control. Am J Trop Med Hyg 55: 125-130.

17. Costa CHN, Pereira HF, Pereira FCA, Tavares JP, Araújo MV, Gonçalves MJO, 1999. Is the household dog a risk factor for American visceral leishmaniasis in Brazil. Trans $R$ Soc Trop Med Hyg 93: 464.

18. Costa CHN, Gomes RBB, Silva MRB, Garcez LM, Ramos PKS, Santos RS, Shaw JJ, David JR, Maguire JH, 2000. Competence of the human host as a reservoir for Leishmania chagasi. $J$ Infect Dis 182: 997-1000.

19. Arias JR, Monteiro PS, Zicker F, 1996. The re-emergence of visceral leishmaniasis in Brazil. Emerg Infect Dis 2: 145-146.

20. Edrissian GH, 1996 Visceral leishmaniasis in Iran and the role of serological tests in diagnosis and epidemiological studies. In Ali Ozcel M, Ziyal Alkass M, eds. Parasitology for the Twenty First Century. CAB International, Wallingford. 63-78.

21. Davies CR, Mazloumi-Gavgani AS, 1999. Age, acquired immunity and the risk of visceral leishmaniasis: a prospective study in Iran. Parasitology 119: 247-257.

22. Nadim A, Javidian E, Tahvildar-Bidruni G, Mottaghi M, Abai MR, 1992. Epidemiological aspects of kala-azar in MeshkinShahr, Iran: investigation on vectors. Iranian J Pub Health 21: 61-72.

23. Nadim A, 1978. Present status of kala-azar in Iran. Am J Trop Med Hyg 27: 25-28.

24. Hamidi AN, Nadim A, Edrissian GH, Tahvildar-Bidruni G, Javadian E, 1982. Visceral leishmaniasis of jackals and dogs in northern Iran. Trans $R$ Soc Trop Med Hyg 76: 756-757.

25. Edrissian GH, Ahanchin AR, Gharachahi AM, Ghorbani M, Nadim A, Ardehali S, Hafizi A, Kanini A, Sarkissian M, Hajaran H, Tahvildari-Bidruni GH, 1993. Seroepidemiological studies of visceral leishmaniasis and search for animal reservoirs in Fars Province, Southern Iran. Iran J Med Sci 18: 99105.

26. Evans DA, Lanham SM, Baldwin CI, Peters W, 1984. The isolation and isoenzyme characterization of Leishmania braziliensis spp. from patients with cutaneous leishmaniasis acquired in Belize. Trans $R$ Soc Trop Med Hyg 78: 35-42.

27. Guarga JL, Lucientes J, Peribáñez MA, Molina R, Gracia MJ, Castillo JA, 2000. Experimental infection of Phlebotomus perniciosus and determination of the natural infection rates of Leishmania infantum in dogs. Acta Trop 77: 203-207.

28. Travi BL, Tabane CJ, Cadena H, Ferro C, Osorio Y, 2001. Canine visceral leishmaniasis in Colombia: relationship between clinical and parasitological studies and infectivity for sandflies. Am J Trop Med Hyg 64: 119-124.

29. Deane LM, Deane MP, 1955. Observações prelimiares sôbre a importância comparativa do homem, do cão e da raposa ( $L y$ calopex vetulus) como reservatórios da Leishmania donovani, em área endêmic de calazar, no Ceará. O Hospital 48: 79-98.

30. Molina R, Lohse JM, Pulido F, Laguna F, Lopez-Velez R, Alvar $\mathrm{J}$, 1999. Infection of sand flies by humans coinfected with Leishmania infantum and human immunodeficiency virus. Am J Trop Med Hyg 60: 51-53.

31. Navin TR, Sierra M, Custodia R, Steurer F, Porter CH, Ruebush TK, 1985. Epidemiologic study of visceral leishmaniasis in Honduras, 1975-1983. Am J Trop Med Hyg 34: 1069-1075.

32. Faris R, Massoud A, El Said S, Gadallah MA, Feinsod FM, Saah AJ, Londner M, Rosen G, 1988. The epidemiology of human visceral leishmaniasis in El Agamy (Alexandria Governate), Egypt: serosurvey and case/control study. Ann Trop Med Parasitol 82: 445-452.

33. Evans TG, Teixeira MJ, McAuliffe IT, Vasconcelos IAB, Vasconcelos AW, Sousa AQ, Linna JW, Pearson RD, 1992. Epidemiology of visceral leishmaniasis in Northeast Brazil. J Infect Dis 166: 1124-1132.

34. Kotkat A, El Daly S, El Daaw A, Barakat R, 1986. Immunological investigation of visceral leishmaniasis among school children in Alexandria. J Egypt Soc Parasitol 16: 449-456.

35. Mauricio IL, Howard MK, Stothard JR, Miles MA, 1999. Genomic diversity in the Leishmania donovani complex. Parasitology 119: 237-246.

36. Costa CHN, Vieira JBF, 2001. Changes in the control program of visceral leishmaniasis in Brazil. Rev Soc Bras Med Trop 34: 223-228.

37. Reithinger R, Teodoro U, Davies CR, 2001. A comparative trial of topical insecticide treatments to protect dogs from bites of sand fly vectors of leishmaniasis. Emerg Infect Dis 7: 872-876. 